\title{
Geoconsolidamenti irreversibili a breve termine
}

\author{
(Irreversible geoconsolidations at brief time)
}

\author{
A. Bfilligi
}

Ricevuto il 25 Agosto, 1975

Riassunto. - Dopo alcune considerazioni sulle geodeidratazioni in uso l'A. sviluppa nuove tecniche, in seguito a neo-orientamenti tcorici in elettrogeosmosi ( $\mathrm{EGO})$.

Introdotto infatti il parametro permeabilità superficiale, presso il catodo, l'A. in tegra con quest'assunto le teorie classiche, pervenendo a neodeduzioni, soprattutto applicative, riguardanti più efficaci consolidamenti dei suoli.

Fondamentale deduzione è che, in condizioni d'applicabilità EGO, l'effetto idroconduttivo si possa pronunciare al catodo in ritardo rispetto a quello EGO.

Tale sfasamento si attua con sufficiente durata, con profilazione iniziale di puro effelto EGO a massima, più o meno accentuato, dando modo di autocondizionare rapidi, intensi effetti consolidanti, di natura irreversibile, ottenendo elettroconsolidamenti a breve termine, a massima intensità, neostrutturazioni a carattere diagenetico in funzione dello sfasamento dei due flussi, idromigratori (al catodo) ionico e neutro, in ordine d'arrivo.

SUmmary. - After some consideration, about the geo-dewatering in use, the $A$. develops new tecniques, following the new theoretical bearings in $\mathrm{EGO}$.

In fact, when the surface permeability parameter is introduced near the cathode, the A. integrates the classic theories with this assumption, attaining new deductions, above all applicable, concerning more efficient consolidations of the layers.

Fundamental deduction is that, in conditions of applicability EGO, the water conductor effect can be pronounced to the cathode in delay, in respect to that of EGO.

Such dephasemont takes place, with initial outlining of pure effect EGO at maximum, more or less accentuated, making it possible to self-condition, rapid and intense consolidated effects of irreversible nature.

Obtaining electroconsolidations at brief terms, at maximum intensity new structures with diagenetic character in function of the dephasement of the two flux, water migrators (by cathode) ionic and neutral, in oriter of arrival. 
1) - Consideriamo un blocco terroso in stato iniziale d'idropressione $H_{o}$, (omogeneo o stratificato), per assoggettarlo a consolidamento. Il procedimento standard $\grave{e}$ il classico pozzo deidratante, reversibile, e a lungo termine. $\Lambda$ fine di tener presente un ordine di grandezza medio di durata consolidante standard " $t$ ", in terreni sedimentari sciolti: $\left(K=10^{-7} \mathrm{~cm} / \mathrm{sec}\right.$; indice vuoti $e=1,5$; stato edometrico $a_{v}=\Delta \varepsilon /$ $\Delta \sigma=3.10^{-4} \mathrm{cmq} / \mathrm{gm} ; Y_{w}=1 \mathrm{gm} / \mathrm{co} ; 1 \mathrm{~m}=$ potenza del test), la durata $t$, o tempo richiesto per un consolidamento del $50 \%$ si valuta in: $c_{v}=k(1+\varepsilon) / a_{r} Y_{\mu},(1$ anno e 3 mesi).

Ora fermo qui restante il criterio generico della geodeidratazione per consolidare, possiamo oggi agire a cospiranti pluri-idroestrazioni, dal pompaggio normale, alla creazione artificiale del vuoto con filtri agliformi (Well-point), in mezzi a facies- sabhiosa, all'elettrodrenaggio osmotico semplice o integrato (elettrochimico) in mezzi a facies colloidali o fini-pulvurulenti (argille, limi, silts).

Vogliamo qui riferirei in particolare al consolidamento terroso elettrosmotico, con dispositivo speciale galvanico a c.c., un elettrobipolo a piani polari paralleli verticali, (realizzabili a slim-holes verticali accostati, in parallelo). I.l dispositivo geoelettrico è per il momento del tipo chiuso, ad esclusione cioè di influenza extranodica, irloneo per i consolidamenti localizzati. Per semplicità il nostro esame analitico è a riferimento unidimensionale, (risultando tuttavia sufficiente ai fini propostici). L'origine delle coordinate è posta ad es. alla testata del catodo, (pozzo tubolare), in superficie orizzontale del suolo, $(x=0)$; $x>0$ verso l'anodo; d'ascissa $(x=a)$, preferibilmente anch'essa tubolare. Lo stato di idrogeopressione: $H(x, t)$ nell'ambito $(0 \leqslant x \leqslant a$; $t>0$ ) soddisferà pertanto, alle equazioni di stato e ai limiti:

$$
\begin{gathered}
\frac{\partial^{2} H}{\partial x^{2}}=c \frac{\partial H}{\partial t}, c=\frac{\Delta \varepsilon / \Delta \sigma}{k(1+\varepsilon)}, \\
H=H_{0},(t=0, c)
\end{gathered}
$$

$c$ è l'inverso del coefficiente di consolidamento standard). Se la differenza di potenziale a c.c. geobipolare è $E$, il campo elettrico interpolare eteronomo sarà $v=E x / a$, (con $a=$ ampiezza eteropolare), da cui discende l'equazione di Helmoltz per la densità d'idroflusso:

$$
q=-k \frac{\partial H}{\partial x}-k_{e} \frac{E}{a}
$$


con $q$ grandezza a due componenti, una relativa all'acqua neutra normale, (a potenziale di velocità), l'altra relativa all'acqua ionizzata EGO, (a potenziale elettrico).

Il dispositivo elettrico è tale, s'è detto, che autoelimina l'infíuenza (qui parassita) extranodica, per cui all'anodo si ha $q=0$, quindi:

$$
\frac{\partial H}{\partial x}+\frac{k_{e}}{k} \frac{E}{a}=0,(x=a) .
$$

Introduciamo a questo punto una permeabilità superficiale finita presso il catorlo verticale tubolare metallico, raggiunto da una migrazione radiale d'idroioni $(+)$, quindi con scarico di idroflusso neutro lungo la superficie cilindrica (v. Fig. 1).
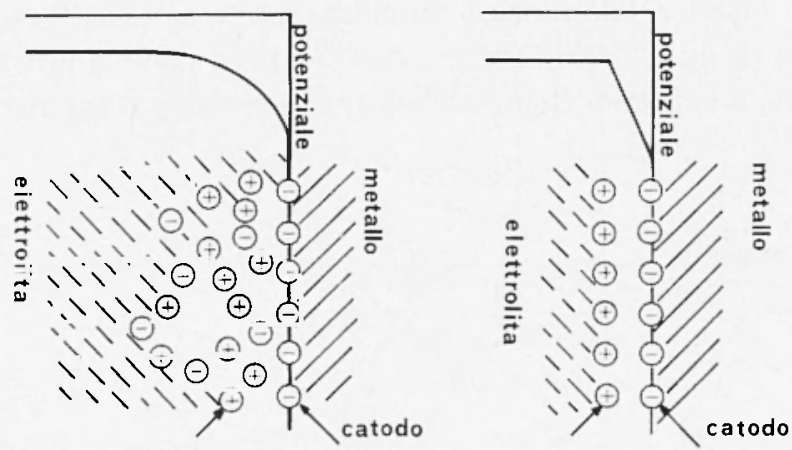

Fig. 1 - Elettroidrosearico lungo le generatrici tubolari catoliche.

Pompando nel pozzo catodico verrì espulsá tale acqua, purchè $H>H_{p}$.

Per $H<H_{p}$, l'acqua verrebbe invece risucchiata nel mezzo con legge lineare.

Se $q_{k}$ è la portata catodica per unità di sezione si avrà:

$$
q_{k}=\chi \cdot\left(H-H_{p}\right)
$$

dove $\psi$ è la permeabilità superficiale: $\chi \chi q_{k} / \Delta H$.

La condizione al catodo risulta per $x==0, q+\ldots .=0$.

Non si deve confondere la contro-direzione di q rispetto alla portata $q k$ con l'inversione di alterazioni processuali, (efletti attennatori 
dapprima, limitativi, spesso inavvertiti come ad es. "l'EGO inversa" o d'acidificazione anodica).

L'acidificazione anodica dopo un certo tempo, $\tau_{a}$, comporta una presenza d'acqua all'anodo, a meno di non interrompere il geocircuito in tempi $\tau<\tau_{a}$, oppure immettere soluzioni alcaline [Na(OH): $1 \mathrm{gm} / 1$; $\mathrm{pH}=\mathrm{g}$ ] o agenti tensiattivi fludex (attivante alcalino).

Per le [4], [5] si ha al catodo $(x=0)$ :

$$
\frac{\partial H}{\partial x}+\frac{\chi}{K}\left(H-H_{p}\right)+\frac{K_{e}}{k} E / a=0
$$

L'equazione [1], con le condizioni [2], [4], [6], definiscono il nostro problema.

Il pozzo estrattivo, libero dalla c.c. a funzionamento normale, presenta la classica falda idrica conoidale fig. 2, se catodizzato si comporta come in fig. 3. Gli anodi, come i catodi, complanari verticali si comportano, a superfici conoidali sempre depressive o aspirative rispettivamente.

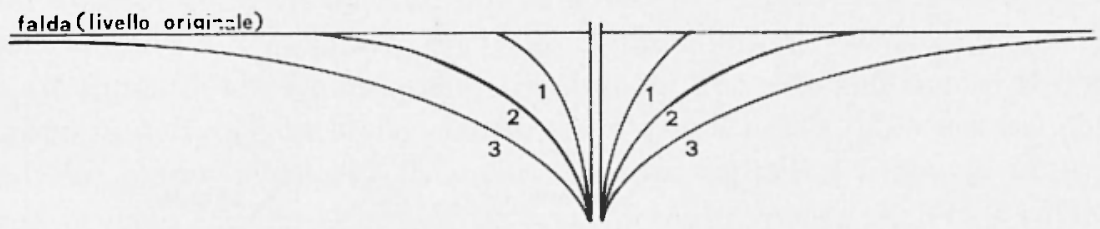

Fig. 2 - Conoidi di abbassamento di falda con solo pompaggio.

1) $-100 \%$ di sabbia; 2) - 50\% di ghiaia e $50 \%$ di sabbia;

3) $-70 \%$ di ghiaia e $30 \%$ di sabbia.

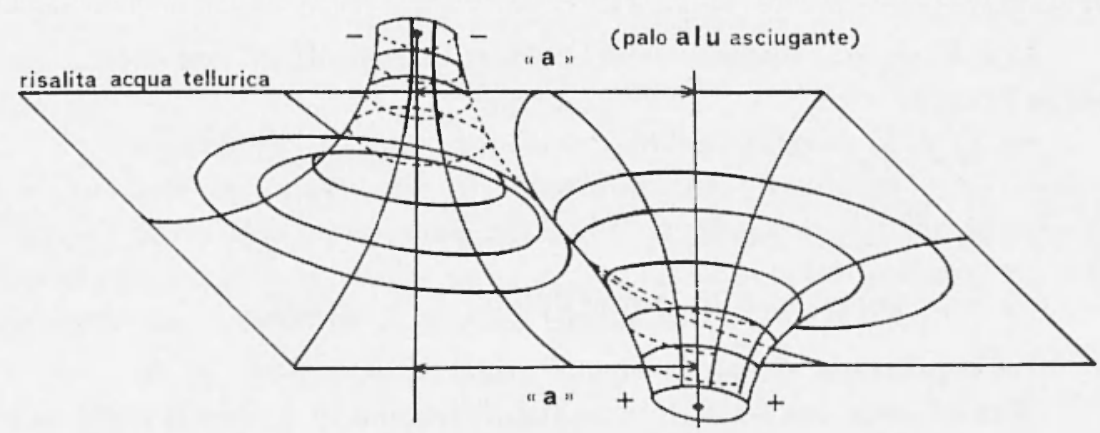

Fig. 3 - Bipolo stretto, spaziatura " $a$ "-interasse conoidi. 
Falde elettroconoidali a linee equipotenziali e di corrente ortogonali appaiono nella fig. 3 .

Il pompaggio, senza immissione di c.c. nel terreno, denuncia semplici conoidi, (figg. 2,3a); le figg. 3, 3b, denunciano invece superfici conoidali complesse, post-immissione di c.c. Il pompaggio integrale si differenzia ancor più, intervenendo la "spaziatura interpolare " (fig. 3a).

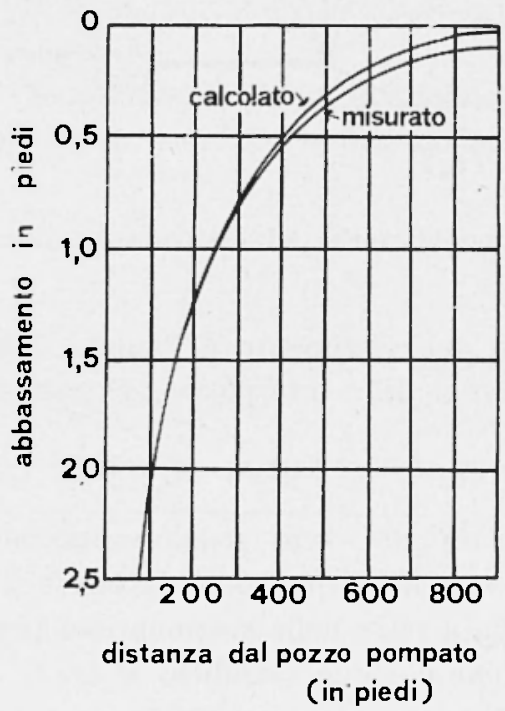

Fig. 3a - Abbassamento falda (calcolato e misurato), massimo in corrispondenza dell'asse del pozzo estrattivo. Simmetria conica di falde depresse in terreni omogenei, a superficie orizzontale del suolo.

Infine si ha il " pompaggio integrale " con immissione galvanica di c.c. (fig. 3b), geobipolo a data spaziatura a eteropolare. A seconda dell'idrosaturazione, il tasso EGO, l'intervento (modalità), l'emungimento catodico, può essere ad acque commiste (neutre ed EGO), oppure separate: EGO e normali.

L'aspirazione a pozzo catodizzato risente nell'interpolarità spaziale della elettrobipolarità; catodo aspiranto, anodo premente; alla simmetria di fig. 3, subentrano asimmetrie (i conoidi extracatodici sono normali).

In "pressioni EGO, in situazione diverse d'idroprelievo catodico" [1], noi abbiamo posto le relazioni che forniscono le trasformate e antitrasformate di Japlace della pressione differenziale $P(x, t)=H(x, t)$ - $H_{o}$, ( $H_{o}$ pressione geoidrostatica iniziale), $P(x, t)$ nulla, o costante al 


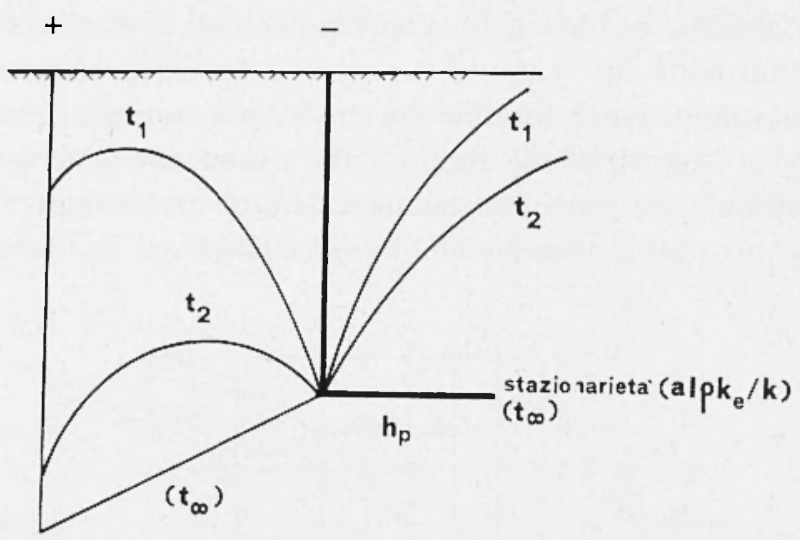

Fig. $3 \mathrm{~b}-$ Conoidi idrici ad elettro-aspirazione catodica.

catodo. Se l'origine del riferimento è l'anodo, spaziatura elettrodica $a$ si hanno 3 spazi (fig. 3c): interpolare, extranodico, extracatodico:

$$
-\infty<0<x<a, \quad a<x<+\infty
$$

Spazio interpolare $(0-a)$ senza espulsione idrocatodica, (a) le falde complesse conoidali evolvono da curve a convessità in alto nella fase transitoria, a rette nella stazionarietà (fig. $3 \mathrm{c}$ ). Questa situazione, senza estrazione d'acqua catodica; $H(x, t)=H_{o}$ è orientativa, di idrotrasferimento.

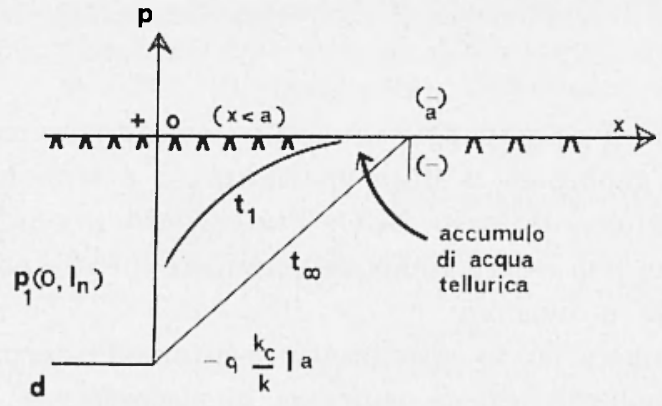

Fig. 3e - Pozzo orientativo eatodico con $H(x, t)=H_{\mathrm{o}}$

Vediamo ora di portare il catodo ad una pressione, pur costante, ma diversa da quella iniziale di ambiente: $H_{o}$. Intanto possiamo scrivere: $P(x, t)=P_{i}$ (componente idraulica) $+P_{e}$ (componente elettrosmotica). 
Introduciamo un valore di pompaggio $P_{a} \neq 0$, (pressione differenziale al catodo): $P_{a-o, t}=P_{a+o, t}$ soggetta a relazione di continuità di $P_{a} \neq 0$ ).

La componente $P_{i}$ pura è data dalla Fig. 3d. Se $P_{o}<0$, ̀̀ relativamente piccola, nel'ambito extra-nodico la pressione differenziale si menterrà negativa.

Al pozzo catodico vige la pressiono $P_{o}$. Se $P_{o}=H_{k}-H_{o}$, è molto grande, la $P_{i}(x, t)$ prevarrà, portando ovunque positivo il valore di $P(x, t)$ (Fig. 3f).

La variazione di curvatura di falde all'altezza dell'infissione $O$ è incidentale, per la presenza ostacolante dell'anodo neutro.

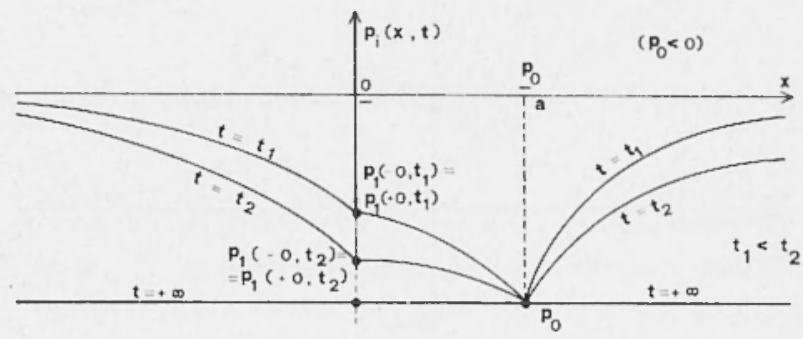

Fig. $3 d-P_{1}$ pura, $E=0, P_{0}<0$.

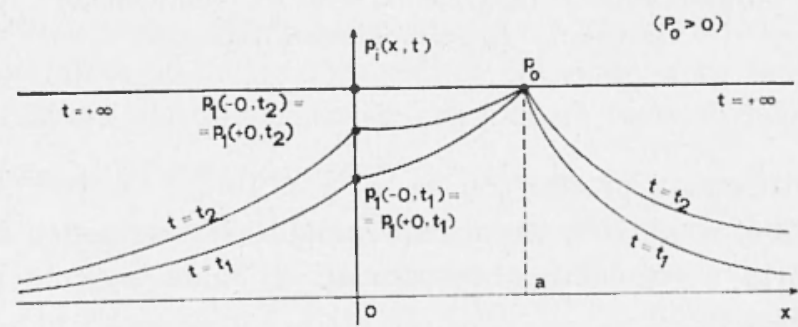

Fig. $3 \mathrm{e}-P_{1}$ pura, $E=0, P_{\circ}>0$.

Le figg. 3d, 3e, mostrano l'abbassamento e l'innalzamento di falda interpolare, a seconda $P_{o} \leqq 0$, abbassamento verso la base catodica, innalzamento in testata del pozzo catodico.

I conoidi d'idroabbassamento rimangono quelli di sempre, salvo appunto risentimenti secondari, come per incontri e distorsioni di schermi neutri, infissi ostacolanti. Emungimenti integrali, (con prevalenza di $\left.P_{i}\right)$, terreni idonei e inidonei all'EGO. 

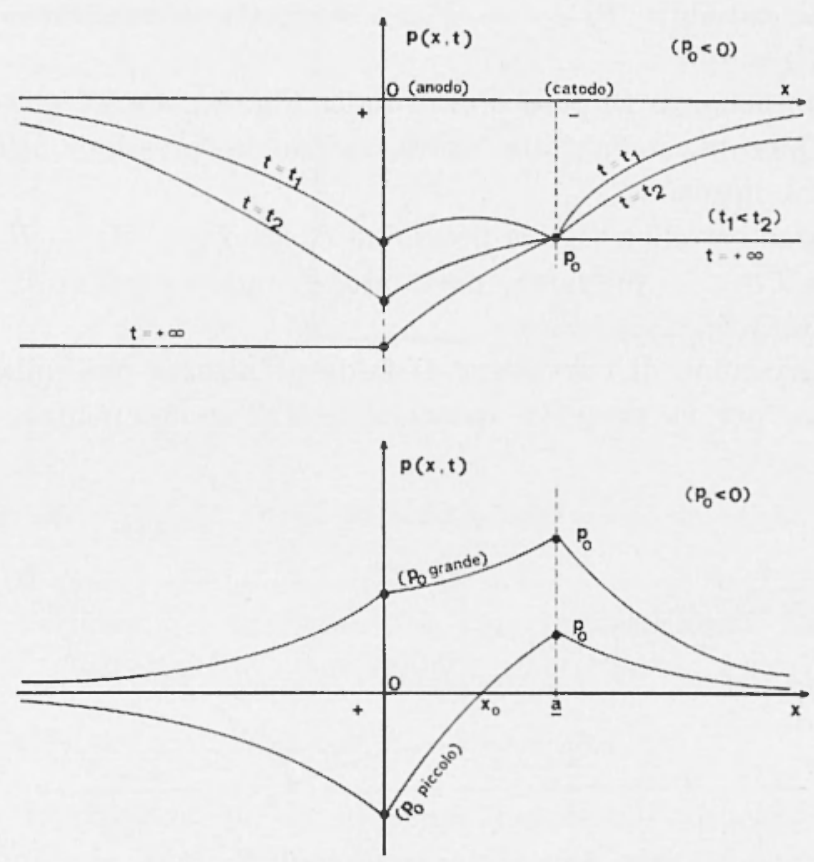

Fig. $3 f$ - Andamento spazio-temporale della pressione differenziale $P(x, t) ; P(x, t)-P_{i}$ (componente Darcy); + $P_{c}$ (componente Helmoltz).

Una situazione pratica (ad es. $k_{e} \varrho=10^{-1}, I=10^{-4}, k=10^{-6}$, $P_{0}= \pm 500, a=100$, su terreno umido elettrocinetico incoerente medio), porta a regolabili abbassamenti di falda, agendo su $E_{e}$ sul $P_{o}<0$, Fig. 3g.

Le depressioni di falda risentono delle profondità polari, per una data loro spaziatura, oltre naturalmente che dei parametri elettrici: $(E, Q)$, e tali superfici passano infine a piani limiti di stazionarietà, in profondità.

Le condizioni ottimali applicative s'ottengono pre-emungendo parzialmente le acque neutre, (non elettro-energizzando ancora il terreno), poi applicando l'EGO, in modo che si promuovano le condizioni della doppia migrazione separata e consecutiva: acqua EGO seguita dalla neutra, (ricordiamo che il moto di quest'ultime $v$, ̀̀ dato da $v=S z(r) \varepsilon E / \pm \pi \eta l)$. 


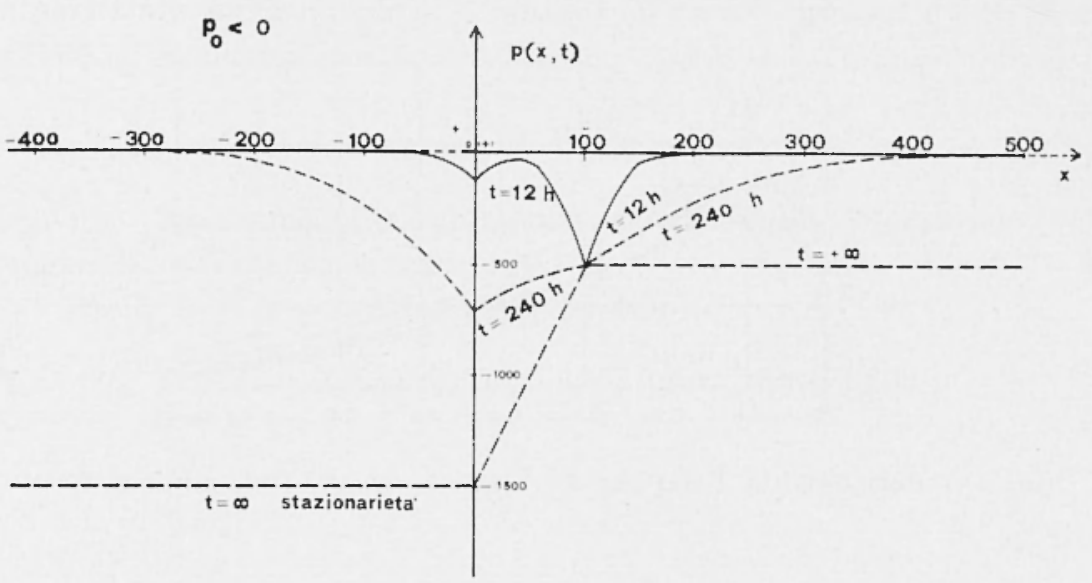

Fig. $3 g$ - Evoluzione dei conoidi misti (anorlici e pozzi catodici).

Mentre l'acqua di Darcy è: $Q_{i}=k_{i} i_{h}$, quella relativa all'EGO ̀̀ $Q_{e}=k_{e} V=k_{e} \varrho I$; (esse denunciano le loro diverse cause), quel che conta è la manovrabilità delle seconde, (per l'avvento della c.c.).

Le permeabilità idrauliche $k$ macroscopiche sono comprese tra la media armonica e la media aritmetica delle permeabilità puntiformi, in mezzi poroso-eterogenei.

Il nostro sistema polare preferenziale è a piani verticali, come accennato, permette tra l'altro d'immettere nel suolo consistenti correnti: il che si realizza con omoelettrodi complanari verticali accostati, per cui la resistenza di cliffusione $\left(R_{\infty}\right)$, si trae da $R_{\infty}^{-1}=\sum_{1}^{n} R_{k}^{-1}$; se gli $R_{k}$ sono uguali, possiedono la stessa resistenza di diffusione unitaria $R_{o}$, si ha $\left(R_{\infty}=R_{0} / n\right)$.

Le $k_{e}$, in funzione del diamstro delle particelle granulari, (parametro principale condizionante $d$ ) sono date in Fig. 4.

Il fascio dei profili (Lomizé), mostra un contenuto " range " variantivo $\left(k_{e}, d\right), d=$ diametro granuli. Diminuendo $d$ il $k_{e}$ aumenta, (rami parabolici, Fig. 4), e a seconda la sostanza, si hanno massimi più o meno a ridosso dell'ordinata " meshs ", piccoli in tutti i casi, $(d=0,1$; 0,15 ;...); avvicinandosi a granulometrie sul $\mathrm{mm}$, il $k_{e}$ scompare (Fig. 4); $d<0,2 \mathrm{~mm}$, comportano $k_{e}$ maggiori.

Le $\left(k, k_{e}\right)$ possono non coesistere; $k$ è variabilissima, $k_{e}$ lo è molto meno, nelle argille $k_{e}$ può risultare però oltre 10.000 volte maggiore del corrispondente $k$ (v. saggi elettro-edometrici). 


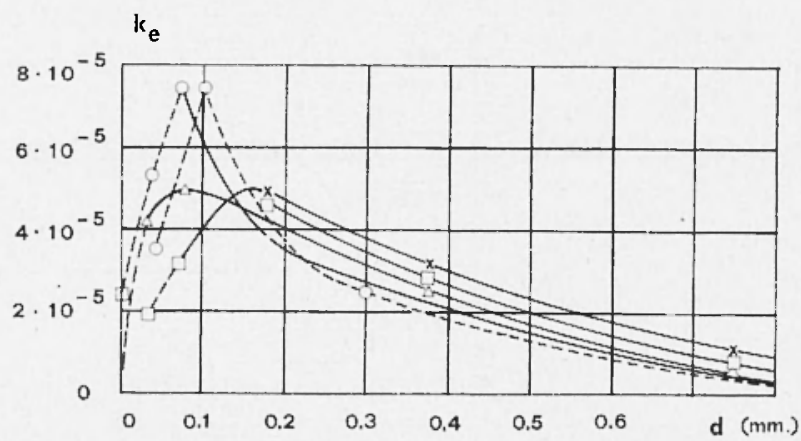

Fiğ. 4 - Permeabilità FGO $\left(k_{e}, d\right)$ : limiti $k_{e}$ (max. 10-4, minimo $10^{-5}$ ).

La superficie esterna del catodo infisso interrompe la idrofalda, e la migrazione radiale EGO scarica un flusso idrico neutro lungo tale superficie. Attivandosi la pompa il flusso laminare lambisce entrambe le superfici, esterna e interna cilindrica; secondo Dachler (1942 Springer), la falda idrica nel pozzo, all'entrata in basso, si stabilisce tangente alla superficie interna. Applicando al catodo fori, tagli, aperture, antintasanti, le linee di iclrofiltrazione scorrono più agevolmente lungo la parete interna, determinandosi spartiacque in seno alla massa fluida ascendente (Bernatzilk).

2) - I parametri, (adimensionali e no), d'impiego più frequente, o principali, in questa teoria, possiamo riassumerli in:

$$
\xi=\frac{x}{a}, \bar{v}=\frac{t}{\tau}, \lambda=a \chi / k ; \%=\varrho_{k} / \Delta H
$$

(azione localizzata al catodo).

Ritornando ora al nostro problema, equazioni [1], condizioni [2], [4], [6], conforme alle [3], [6], la idrogeopressione $H$ si può scomporre in due componenti:

$$
H_{p}+\left(H_{0}-H_{p}\right) \varphi \quad ; \quad H_{p}+-\frac{k_{e}}{k} \psi
$$

idroconduttiva pura $(E=0)$, elettrosmotica pura: $H_{0}=H_{p}$; noi abbiamo introdotto in entrambe tali componenti le funzioni adimensionali $\varphi(\xi, \vartheta), \psi(\xi, \vartheta)$, moltiplicatori che dipendono dalle varie costanti attraverso un neo-rapporto, che introduciamo ora, di permeabilità 
superficiale finita $\lambda$, (la $\varphi$ si riferisce all'effetto idroconduttivo, la $\psi$ all'EGO). Poniamo cioè:

$$
\lambda=a \chi / k_{i}
$$

rapporto di permeabilità numerico (permeabilità superficiale per lunghezza, e i(lraulica spaziale).

Per $E=0$ (puro effetto igroconduttivo):

$H_{i}=H_{p}+\left(H_{0}-H_{p}\right) \varphi ;$ per $H_{0}=H_{p}$ (puro effetto EGO):

$$
H_{e}=H_{p}+\frac{k_{e}}{k} E \psi
$$

Non si tratta però di vero "puro effetto EG() ", a meno di conferire un significato convenzionale, perchè in sostanza la [1] contiene anche l'effetto igroconduttivo. Si può intendere, a rigore, più che un "puro effetto EGO", parte dell'effetto integrale proporzionale a $E$. $\mathrm{E}$ facile fin d'ora intuire ehe il neo-parametro numerico $\lambda$ non potri che comparire nelle funzioni moltiplicatrici $\varphi, \psi$.

In "Aspetti diversi dell'EGO trans (1958) " introducemmo già un coefficiente di proporzionalità $\lambda$ relativo alla pressione al catodo, $H_{k}=\lambda\left(\int_{0}^{t} q_{k} \mathrm{~d} t-\int_{0}^{t} \chi \mathrm{d} t\right),\left(\chi=\right.$ velocità di prelievo d'acqua, $q_{k}$ portata catodica per unità di sezione, $(\lambda \chi)$ velocità di caduta della pressione idrostatica col pompaggio al pozzo catodico). Il neo-coefficiente $\lambda$ ̀̀ dunque il rapporto tra la variabilitì della pressione catodica e il flusso catodico, (dell'ordine di grandezza appunto del rapporto tra la pressione catodica e la quantitì d'acqua contenuta in una sua sezione unitaria).

Con catodo prismatico, sezione rettangolare di spessore $d$, altezza $l$, gravitì $g$, tale rapporto si può brevemente indicare con $(g \cdot l / d)$. In realtà $\lambda$ è più piceolo: il catodo non è sempre colmo d'acqua, $l$ dovrà perciò esser sostituito con un valore medio $l$, dell'altezza della colonna d'acqua nel pozzo catodico: $(l / l<1,=\alpha<1 / 2$, e anche meno). Si considera poi, normalmente, la pressione in fondo, invece è da prevedere una merlia, quindi altro fattore 1/2. La idropressione si comunica nel terreno circostante attraverso for i praticati sul catodo (alla base, per un certo tratto). Indicando con $\beta=s^{\prime} / s$ il rapporto tra superficie forata e totale, si perviene al fattore $\beta$. Infine l'idropressione e le sue variazioni si trasmettono comunque imperfettamente al suolo circo- 
stante, quindi ulteriore riduzione $\gamma$, frazione dell'unità. Per $\lambda$ si perviene all'ordine di grandezza $(\lambda \sim 1 / 2 \alpha \beta \gamma g l / d),(1 / 2 \alpha \beta \gamma$ in una grande varietà di casi $=1 / 100)$. Si noti ancora che la $H$ è una grandezza, che insieme a $k$ e formano la base della nostra teoria.

Se al tempo $t$, preleviamo una certa quantità d'acqua, provocando una depressione catodica $\Delta H$, si avrà $\lambda \psi=\Delta H \delta(t-E)$ dove $\delta$ indica la funzione impulsiva di Dirac.

La $H$ ì sostanzialmente legata alla quantità d'acqua contenuta nel terreno per uniti di volume: $H-H_{0}=\left(w-w_{0}\right) / k c$, una relazione a carattere puramente formale. La $H$ è stata in fondo introdotta per trascrivere l'effetto Darcy dell'idroflusso:

$$
\vec{q}=-k \operatorname{grad} H--\frac{1}{c} \operatorname{grad} w,
$$

non coinvolge l'effetto Holmoltz (EGO), dato che in esso $H$ non compare. La $\vec{q}$ fissa il significato di $c$, e insieme all'equazione di diffusione $(\Delta H=c \partial H / \partial t)$, si determinano carattere e avanzamento fenomenico. La $c$ si può determinare sperimentalmente con i vari dispositivi, come quello dei due cilindri polari concentrici. In ogni caso se la determinazione di $c$ si compie nella stazionarietà, ̀̀ necessario risalire al grad $w$, se si compie nelle transitorietà, basta determinare sperimentalmente i tempi caratteristici, da essi ricavare la $c$. L'equazione che dà $\vec{q}$ mostra che la presenza di $k$ è puramente formale, ed è strettamente legata alla definizione di $H$, (nelle formule dove non compaiono pressioni non appare nemmeno $k$ ). Dopo queste considerazioni possiamo chiarire il significato di $\gamma$ che appare in:

$$
\lambda=g-\frac{l}{d} \alpha \beta \gamma, \quad\left(\alpha=1 / 4, \beta=s^{\prime} / s\right)
$$

In quest'eguaglianza ì implicita l'ipotesi che la pressione effettiva al catodo $\bar{H}_{k}$ è proporzionale alla pressione formale delle immediate vicinanze del catodo. Se si potessero identificare le 2 pressioni, si avrebbe $\gamma=1$, ma in realtà non sono uguali.

Veramente in $\gamma$ si è anche conglobato l'effetto relativo alle dimensioni finite del catodo. Conviene aggiungere ancora in $\lambda$ un altro fattore dispersivo $\delta$ che tiene conto di tali dimensioni finite: esso non 
sarà molto più piccolo di 1 , se l'ampiezza interelettrodica è dell'ordine delle dimensioni degli elettrodi $\lambda=g \frac{l}{d} \alpha \beta \gamma \delta$.

Ritornando alle due pressioni $H_{k}$ e $\bar{H}_{k}, H_{k}=w_{k} / k c,\left(w_{k}=\right.$ unità del terreno nella zona catodica), allora

$$
\gamma=H_{k} / \bar{H}_{k}=w_{k} / k c \bar{H}_{k}, \quad \text { o } H_{k}-\gamma \bar{H}_{k} .
$$

Il rapporto $\gamma$ cost. equivale ad ipotizzare che ogni qualvolta il terreno saturo viene a contatto coll'atmosfera, si stabilisce subito un equilibrio tra la pressione esterna e l'umidità del suolo a contatto, da cui la proporzionalità $H_{k}, \bar{H}_{k}$.

Sostituendo infine le [8] in [1], eseguendo varie trasformazioni, si perviene ai seguenti due problemi riguardanti le $\varphi, \psi$ :

1) determinare nella striscia di terreno $(o \leqslant \xi \leqslant a, \vartheta>0)$, una funzione $\varphi$ che soddisfa alle seguenti condizioni:

$$
\frac{\partial^{2} \varphi}{\partial \xi^{2}}=\frac{\partial \varphi}{\partial t}
$$

e alla condizione iniziale

$$
\varphi=1 \quad \text { per } \vartheta=0
$$

in più al contorno:

$$
\frac{\partial \varphi}{\partial \xi}-\lambda \varphi=0,(\xi=0) ; \frac{\partial \varphi}{\partial \xi}=1,(\xi=1)
$$

2) determinare, nella stessa striscia, una $\psi$ che soddisfi alla:

$$
\frac{\partial^{2} \psi}{\partial \xi^{2}}=\frac{\partial \psi}{\partial t}
$$

alla condizione iniziale

$$
\psi=0 \text { per } \vartheta=0
$$

al contorno:

$$
\frac{\partial \psi}{\partial \xi}-\lambda \xi+1=0,(\xi=0) ; \quad \frac{\partial \psi}{\partial \xi}+1=0,(\xi=1)
$$


Ricavate le $\varphi, \psi$ si avrà per la portata globale al catodo d'emungimento idrico:

$$
q_{k}=-\frac{k}{a}\left(H_{\mathrm{o}}-H_{p}\right) \lambda \varphi(0, \vartheta)+\frac{h_{c}}{a} E \lambda \psi(o, \vartheta)
$$

Per risalire a $\varphi, \psi$, ci serviamo delle trasformate di Laplace: $\bar{\varphi}, \vec{\psi}$, le trasformate rispetto a $\vartheta$ siano:

$$
\bar{\varphi}(\xi, s)=\int_{0}^{\infty} e^{-s \vartheta} \varphi \mathrm{d} \vartheta ; \quad \bar{\psi}(\xi, s)=\int_{0}^{\infty} e^{-\hat{-0 \vartheta}} \psi \mathrm{d} \vartheta
$$

(gli apici sulle $\varphi, \psi$, denotano le derivazioni rispetto a $\xi$ ), cosicchè la trasformazione dei due problemi porta ai seguenti due altri problemi:

3) determinare una $\bar{\varphi}$ che nell'intervallo $0 \leqslant \xi \leqslant 1$ soddisfa la:

$$
\vec{\varphi}^{\prime \prime}-s \bar{\varphi}^{\prime}+1=0
$$

le condizioni ai limiti:

$$
\left.\bar{\varphi}^{\prime}-\lambda \varphi=0,(\xi=0) ; \bar{\varphi}^{\prime}=0, i^{\prime}=1\right)
$$

4) determinare una funzione $\bar{\psi}$ che soddisfa nell'intervallo $(0 \leqslant \xi \leqslant 1)$ alla:

$$
\bar{\psi}^{\prime \prime}-s \psi^{\prime}=0
$$

e alle condizioni ai limiti:

$$
\bar{\psi}^{\prime}-\lambda \bar{\psi}+s^{-1}=0 ; \xi=0 ; \bar{\psi}^{\prime}+s^{-1}=0 ; \xi=1
$$

Le soluzioni di queste equazioni differenziali ordinarie si trovano coi metodi standard dell'analisi. Esse risultano:

$$
\begin{gathered}
\bar{\varphi}=s^{-1}-\frac{\lambda}{s \Delta s} \operatorname{ch}(1-\xi) \sqrt{\xi} \\
\bar{\psi}=\frac{1}{s \Delta s}\left\{\operatorname{ch}(1-\xi) \sqrt{s}-\operatorname{ch} \xi \sqrt{s}-\lambda \frac{\sin \xi \sqrt{ } s}{\sqrt{s}}\right\}
\end{gathered}
$$

ove si ì posto:

$$
\Delta(s)=\sqrt{s} \cdot \operatorname{sh} \sqrt{s}+\lambda \operatorname{ch} \sqrt{s}
$$


Si deve ora passare alle antitrasformate delle [16] e [16']. Si tratta di funzioni analitiche meromorfe, i cui poli si trovano sull'asse immaginario negativo, e il cui comportamento all'infinito è tale che si può applicare il noto metodo dei residui. I poli oltre a $s=0$ per la [16], s'ottengono risolvendo l'equazione: $\Delta(s)=0$. Indicando le soluzioni di queste equazioni con $\left(s_{n}\right)$ e posto: $\left(s_{n}=-\mu^{2}{ }_{n}\right)$, si perviene all'equazione fondamentale:

$$
\mu \tan \mu=\lambda
$$

Le soluzioni della [18] si possono ottenere graficamente, o trovando l'intersezioni della funzione $y=x \tan x$ con la retta $y=\lambda$, o quella della funzione $y=\operatorname{ctg} x$ con la retta $y=x / \lambda$, o col metorlo di Newton per avere valori più precisi. Si trova cosi che le soluzioni formano una successione $\mu_{n},(n=0,1, \ldots)$, con:

$$
n \pi \leqslant \mu_{n} \leqslant\left(n+\frac{1}{2}\right) \pi
$$

tale che per $\lambda$ che varia tra 0 e $\infty$, le $\mu_{n}$ variano tra: $n \pi$ e $\left(n+\frac{1}{2}\right) \pi$. Per $n \gg \frac{\lambda}{\pi}$, si ha asintoticamente:

$$
\mu_{n}=n \pi+\frac{\lambda}{n \pi}
$$

Inoltre, in luogo delle [18], [18'] si hanno le seguenti relazioni:

$$
\begin{aligned}
\tan \mu_{n}= & \frac{\lambda}{\mu_{n}} ; \cos \mu_{n}=(-1)^{n} \mu_{n} / \sqrt{\lambda^{2}+\mu^{2}{ }_{n}} ; \\
& \operatorname{sen} \mu_{n}=(-1)^{n} \lambda / \sqrt{\lambda^{2}+\mu^{2}{ }_{n}}
\end{aligned}
$$

Per calcolar' i residui occorre anzitutto l'espressione di $s_{n} \Delta^{\prime}\left(s_{n}\right)$ che, utilizzando la [20], risulta:

$$
s_{n} \Delta^{\prime}\left(s_{n}\right)=-\frac{1}{2}(-1)^{n} \cdot\left(\mu^{2}{ }_{n}+\lambda^{2}+\lambda\right) / \sqrt{\lambda^{2}+\mu^{2}}
$$

Mediante le [20], [21], si trovano le espressioni per i residui relativi ai poli $s_{n}$, delle $\bar{\varphi}$ e $\bar{\psi}$ rispettivamente:

$$
\begin{gathered}
2(-1)^{n} \cos \mu_{n}(1-\xi) \frac{\lambda \sqrt{\mu^{2} n+\lambda^{2}}}{\mu_{n}\left(\mu^{2}{ }_{n}+\lambda^{2}+\lambda\right)} \\
2 \sqrt{\mu^{2}{ }_{n}+\lambda^{0}} \cdot \cos \mu_{n}(1-\xi) \frac{\sqrt{\mu^{2}{ }_{n}+\lambda^{2}}-(-1)^{n} \mu_{n}}{\left(\mu_{n}^{2}+\lambda^{2}+\lambda\right) \mu^{2}{ }_{n}}
\end{gathered}
$$


$\Lambda$ questi si aggiunge ancora, per la sola $\psi$, il residuo proveniente dal polo $s=0$ che si calcola immediatamente uguale a $(-\xi)$.

Introducendo le seguenti:

$a_{n}=\frac{2 \lambda \sqrt{\mu^{2}{ }_{n}+\lambda^{2}}}{\mu_{n}\left(\mu^{2}{ }_{n}+\lambda^{2}+\lambda\right)} ; b_{n}=2 \sqrt{\mu^{2}{ }^{2}+\lambda^{2}} \frac{\sqrt{n^{\ell^{2}}+\lambda^{2}}-(-1)^{n} \mu^{2}{ }_{n}}{\mu^{2}{ }_{n}\left(\mu^{2}{ }_{n}+\lambda^{2}+\lambda\right)}[22]$

si ha per le $\varphi, \psi$, (che risultano serie convergenti adatte per il calcolo numerico):

$$
\left\{\begin{array}{l}
\varphi(\xi, \vartheta)=\sum_{n=0}^{\infty}(-1)^{n} a_{n} \cos \mu_{n}(1-\xi) e^{-\mu_{n}^{2} \vartheta} \\
\psi(\xi, \hat{v})=-\xi+\sum_{n=0}^{\infty} b_{n} \cos \mu_{n}(1-\xi) e^{-\mu^{2} \vartheta}
\end{array}\right.
$$

Sostituite nelle [8], le [23] permettono di valutare finalmente la (H) in forma esatta, a tre permeabilità $\left(k, k_{c}, \psi\right)$ :

$$
\begin{gathered}
H=H_{p}+\left(H_{o}-H_{p}\right) \sum(-1)^{n} a_{n} \cos \mu_{n}(1-\xi) e^{-\mu_{n}^{2} \vartheta}+ \\
+\frac{k_{e}}{k} E\left[-\xi+\sum b_{n} \cos \mu_{n}(1-\xi) e^{-\mu_{n}^{2} \vartheta}\right]
\end{gathered}
$$

Sostituite nella [13], le [23] permettono analogamente di risalire a $q_{k}$, (lunque abbiamo rigorosamente calcolate pressioni e portate, post-assunzione del neo-parametro permeabilita superficiale, le cui ripercussioni ci hanno permesso nuovi sviluppi tecnici.

L'elettrodeidratazione del mezzo spazio - temporale, interpolare, e extra-polare anodica - risente dell'incidenza del $\tau$ a seconda i tempi di consolidamento: $\tau / n, \tau, n \tau$, pur cercando di limitarsi ai tempi più produttivi $(\tau / n)$, che si raggiungono molto prima di quanto non sia stato affermato lino ad oggi conforme del resto all'esperienza sul terreno.

L'cmungimento consolidante richiede infatti percentuali limitate di deidratazioni, (per $t \ll \tau$ ), a cominciare dall'acqua interstiziale, che tra l'altro scoesizza, indebolisce il modulo dinamico della roccia. ed è quella di saturazione, la cui eliminazione anche parziale, causa immerliate insorgenze di menischi di bondaggio, in genere tesissimi, tra $\mathrm{i}$ granuli solidi. 
Come si verle per $\vartheta \rightarrow \infty$ la $\varphi=0$, la $\psi=-\xi$, il che corrisponde al fatto che $H$ tende al suo valore stazionario: $\left(\pi_{n}-\frac{k_{e}}{k} E \xi\right)$, corrispondente al suo iter lineare; pressione $H_{p}$ al catodo $\left(H_{p}-\frac{l_{e}}{k} E\right)$, all'anodo. Per $\bar{v}=1, t=\tau$, praticamente si possono trascurare tutti i termini delle serie tranne il primo.

Ora mentre col salire di " $n$ ", gli esponenti diventano poco sensibili a $\lambda$ (v. [18']), la $\mu_{\mathrm{o}}$ dipende invece fortemente cla $\lambda$.

Precisamente per alti valori della permeabilità superficiale $\mu_{o}$ sarà vicino a $\pi / 2$, quindi si potrà raggiungere il regime in un tempo d'ordine di grandezza di solo qualche multiplo del tempo caratteristico o di consolidamento $\tau$.

Ancora pegrio se la permeabilità superficiale ì tanto bassa che il rapporto $\lambda$ risulta piccolo in confronto coll'unità $(\lambda=a \chi / k<1)$.

In questo caso anche $\mu_{0}$ risulterà piccola, (dell'ordine $\sqrt{\lambda}$ ), e l'influenza del primo termine $(n=0)$ si farì sentire per un tempo $\frac{\tau}{\mu_{0}{ }^{2}}$ ciò̀ $\frac{\tau}{\bar{\lambda}}=\frac{a}{\chi}$, col risultato che per molto tempo lo stato di regime non potrà essere raggriunto.

Per precisare questo comportamento per bassi valori di $\lambda$, si ha:

$$
\mu_{\mathrm{o}}=\sqrt{\lambda}, a_{\mathrm{o}}=1, b_{\mathrm{o}}=\frac{1}{2} ;
$$

e per $\vartheta \leqslant 1$ si derivano per $p, \psi$ ottenendo:

$$
\varphi=\cos \sqrt{\lambda}(1-\xi) e^{-\lambda . \lambda}, \psi=-\xi+\frac{1}{2} \cos \sqrt{\lambda}(1-\xi) e^{-\lambda \vartheta}
$$

In altre parole, in queste condizioni, si ha per la itrogeopressione $H$ :

$H=H_{p}-\xi \frac{l}{i}+\left\{H_{0}-H_{p}+\frac{1}{2} \frac{k}{i} E^{\prime}\right\} \cos \sqrt{\lambda}(1-\xi) e^{-\lambda . \vartheta}$

Il termine tra parentesi rappresenta una grandezza che per tempi $\leqslant \tau / \lambda=a / \chi$, ̀̀ dello stesso ordine del primo.

Si perviene al seguente $1^{\circ}$ teorema:

Fino a quando $t \ll \tau / \lambda(=a / x)$, allora l'effetto idroconduttivo non $\dot{e}$ ancora efficiente, mentre quello EGO sposta l'acqua dall'anodo al catodo, ove essa si raccoglie (o viene estratta). 
Ancora una volta la permeabilità superficiale è, unitamente alla spaziatura polare, fortemente condioionante. Siccome poi in pratica ¿ importante che il fenomeno avvenga in tempi $(<\tau)$, ì dunque necessario aumentare possibilmente la permeabilita superficiale. Ne segue il corollario: nella unidimensionalitì per diminuire $\tau / \lambda$, si può ridume l'ampiezza interelettrodica; nella situazione reale tridimensionale occorre aumentare la superficie dei catodi cilindrici, (la resistenza olmica diminuisce col loro diametro $\varnothing \mathrm{su} l=10 \mathrm{~m}$, essa dà $10 \Omega$, per $\varnothing=10 \mathrm{~cm}$; scende a $6 \Omega$, per $\varnothing=60 \mathrm{~cm})$. Lielettrodologia migliore ì ad elettrodi accostati, anodi in serie, grossi catorli.

La separazione dei due flussi dipenderà per una data entità di pompaggio al pozzo eatorlico sia dalle aliquote elettrogeocinetiche, che di saturazione, (queste ultime pre-rettificabili); la estrattivita iniziale più rapicla possibile. Per lo meno superfluo, nonchè antieconomico, appare quindi spingere il processo post-massimo flusso EGO, coprendo questa fase temporale il massimo e piu efficace consolidamento, (attuabile unicamente in tale periorlo). So l'emungimento avviene ad acque commiste, non potranno più verificarsi che deboli consolidamenti e a lungo termine, come accarle attualmente.

Nel periodo di flusso iniziale isolato EGO, il dielettrico terroso ì serle di pressioni mecaniche, azioni ponderomotrici d'elettrostrizioni, di elettrocapillarità, tutti effetti inesistenti se il flusso EGO ì commisto all'idraulico. E per questo che le prassi EGO entrano in una nuova fase d'insospettato rendimento globale.

3) - Ritorniamo all'anclamento di $\varphi, \psi$, in funzione del tempo, sull'morlo $\xi=1$ e sul catorlo $\xi=0$; si vede che su quest'ultimo, in virtù della [13], la portata è proporzionale alla differenza $\left(H-H_{p}\right)$.

Quindi tutto ciò che vale sul comportamento della pressione sul catodo, si trasferisce immediatamente alla portata, pur ricordando che la portata differisce da questa pressione per un semplice fattore.

Dalle [23] si ricavano, per le pressioni, sull'anodo:

$\varphi(1, \vartheta)==\sum_{0}^{\infty}(-1)^{n} a_{n} e^{-\mu^{2} n \vartheta} \quad ; \quad \psi(i, \vartheta)=-1+\sum b_{n} e^{-\mu_{n}^{2} \vartheta}$

sul catodo:

$$
\varphi(0, \vartheta)=\sum \alpha_{n} e^{-\mu^{2} n \vartheta} \quad ; \quad \psi(0, \vartheta)=(-1)^{n} \beta_{n} e^{-\mu^{2} n \vartheta}
$$


ove si i posto: per $\alpha_{n}, \beta_{n}$ :

$$
\alpha_{n}=\frac{2 \lambda}{\mu^{2}{ }_{n}+\lambda^{2}+\lambda}, \beta_{n}=2 \frac{\sqrt{\mu^{2}{ }_{n}+\lambda^{2}}-(-1)^{n} \mu_{n}}{\mu_{n}\left(\mu_{n}{ }^{2}+\lambda^{2}+\lambda\right)}
$$

Ja $\varphi$ diminuisce dal valore iniziale 1 a 0 , piu rapidamente al catorlo che non all'anorlo, avendosi nel primo caso una serie di segno costante, nel secondo una serie di segno alterno.

Questo fatto comisponde al libero deflusso dell'acqua dal livello $H_{o}$ al livello $H_{p}$ al catodo, supposto che all'anodo tale deflusso non abbia luogo. Per la $\psi$ si hanno effetti diversi all'anodo (analogamente alla diminuzione continua di $p$ ); si passa dal valore iniziale $\psi=0$ al finale $\psi=-1$.

Al catorlo valori iniziale e finale coincidono e sono uguali a $\psi=0$. Inoltre la serie $\psi(0, \vartheta)$, non solo d̀ a segno alterno; col crescere di $n$ i termini con segno negativo (n dispari) per la presenza di $(-1)^{n}$ nella formula di $\beta_{u}$, acquistano maggior importanza di quelli con segno positivo ( $n=0$, o pari). Questo comportamento, inseme ad ulteriori considerazioni, sull'andamento iniziale della $\psi(0, \vartheta)$, mostra che la $\psi$ si porterà all'inizio fino ar un massimo, per poi decrescere a zero.

Il che corrisponde al fatto ( $2^{\circ}$ teorema):

data la permeabilità superficiale finita, inizialmente lacqua al catodo, sotto l'influenza del campo, affuirà più rapidamente di quanto non possa defluive attraverso la superficie del catodo, fino a raygiungere un massimo; dopodichè l'afflusso, per l'effetto idrogeoconduttivo contrario, diminuirà e scenderà sotto il livello d'acqua defluente attraverso la superficie.

Si evidenzia, nell'idrogeomigrazione, la possibilità di uma separazione delle acque temporale, elettrica prima, idraulica poi, permettente una incisica azione consolidante, che si svolge nella fase elettrica. Il massimo puro EGO verri raggriunto tanto prima quanto più grande sarà 2. Per l'azione di elettroidrosmalicamento si aprono più vaste porosita a immerliate forti compattazioni per il carico idroponderale indenne, le acque interstiziali e fase solida. I'effettivo fenomeno naturalmente non si presenterì con l'andamento delineato, dato che in esso se ne sovrappongono due: se dominante i l'EGO, la q $k$ accuseri un profilo simile a quello descritto, con la differenza che il valore iniziale non sarà dato da 0 , ma da $\lambda\left(H_{0}-H_{p}\right) k / a$, a massimo anticipato.

Se invece preslomina l'efletto idroconduttivo, la $q k$ diminuirà continumente ral valore sopracitato a zero. I casi intermedi rassomi- 
glieranno più o meno ad uno dei due estremi, a seconda che si abbia:

$$
k E / k\left(H_{0}-H_{p}\right) \gtrless 1
$$

ed è relativamente facile separare nei profili i due addendi. Comunque si dispone d'altri mezzi, cruciali, per segnare, nel tempo processuale, l'arrivo delle acque libere per spinta Darcy dopo le acque elettrogeosmotiche per spinta elettrica.

İ nella $1^{\text {ia }}$ fase del profilo $\left(q_{h}, t\right)$ che l'EGO libero (acqua più veloce) può operare più intensamente, l'acqua più veloce EGO apparendo isolata prima dell'altra normale, non legata, elettricamente neutra, (non escludendo in pratica, s'è detto, predesaturazioni del suolo senza c.c., ed altri interventi per metterci inizialmente in situazione di non prevalenza o simultaneità dei flussi Darcy). La durata del processo migratorio della componente iniziale pura EGO dà modo, ripetiamo, ad una più intensa, rapida, diversa compattazione geomecanica, attivandosi contemporaneamente processi elettrochimici per ancor più saldi e diagenetici neo-consolidamenti.

Alle già accennate variazioni volumetriche d'elettrostrizione, si aggiungono succhiamenti del dielettrico verso punti ove il c.e. è più intenso: effetti triboelettrici si manifestano nello strofinio d'arlattamento dei depositi: insorgono vere e proprie neostrutture di cui parleremo a parte.

Passiamo a derivare le formule approssimate, per le grandezze [27], relative ai tempi $\vartheta$ molto piccoli $(\vartheta \ll 1)$. Com'è noto il comportamento d'una funzione per $\vartheta \rightarrow 0$, dipende essenzialmente da quello della sua trasformata per $s \rightarrow \infty$; ora si ha per grandi valori di $s$ :

$$
\operatorname{sh}(s)-\operatorname{ch}(s) \sim \frac{1}{2} e^{\sqrt{s}}
$$

per cui dalle [16], [16'], si ottiene per così grandi valori di $s$ :

$\bar{\varphi}(\vartheta, s)=s^{1}-\lambda / s(\sqrt{s}+\lambda) ; \bar{\psi}(\theta, s)=1 / s(\sqrt{s}+\lambda)$

Le antitrasformate della [29] daramno il comportamento iniziale dei valori di $\varphi, \psi$ sul catodo.

A questo scopo anzitutto occorre determinare l'antitrasformata di $\lambda / s(\sqrt{s}+\lambda)$, partendo dalla nota formula: 


$$
L^{-1}\left(\sigma^{-1} \rho, 2 \mu \sqrt{\sigma}\right)=\frac{2}{\sqrt{\pi}} \int_{x / \sqrt{\theta}}^{\infty} e^{-\varkappa^{2}} \mathrm{~d} z
$$

( $L^{-1}$ è l'antitrasformata). Moltiplicando per $2 \lambda e^{-2 x \lambda}$ e integrando rispetto ad $x$ tra 0 e $\infty$ si ottiene:

$$
L^{1}-\frac{\lambda}{s(\sqrt{s}+\lambda)}-\frac{4 \lambda}{\sqrt{\pi}} \int_{0}^{\infty} e^{-2 x \lambda} \mathrm{d} x \int_{x / \sqrt{\vartheta}}^{\infty} e^{-z^{2}} \mathrm{~d} z-1-\frac{e^{2 \cdot \Omega}}{\sqrt{\pi}} \int_{\lambda \sqrt{\vartheta}}^{\infty} e^{-y^{2}} \mathrm{~d} y
$$

Da questa si trae immediatamente, per $\vartheta \ll 1$ :

$$
\begin{gathered}
\varphi(0, \vartheta)=\frac{2}{\sqrt{\pi}} e^{-\lambda^{2} \vartheta} \int_{\lambda \sqrt{\vartheta}}^{\infty} e^{-y^{2}} \mathrm{~d} y \\
\psi(0, \vartheta)=\lambda^{-1}\left\{1-\frac{2}{\sqrt{\pi}} e^{-\lambda^{2} y} \int_{\lambda \sqrt{\vartheta}}^{\infty} e^{-y^{2}} \mathrm{~d} y\right\}
\end{gathered}
$$

Le [30] mostrano che la $\varphi(0, \vartheta)$ decresce, mentre la $\psi(0, \vartheta)$ aumenta inizialmente, il che completa considerazioni già fatte.

Inoltre la velocità con cui $\psi$ aumenta dipende da $\lambda$ : la presenza di $\left(9 \lambda^{2}\right)$ fa supporre che la posizione del massimo per grandi $\lambda$ dovrebbe trovarsi nella zona $\vartheta \sim \lambda^{-2}, t=\tau / \lambda^{2}$; cioè esso varierà fortemente con il "rapporto di permeabilità " $\lambda$, mentre sarà qui poco dipendente dalla ampiezza polare $a$.

Probabilmente per bassi $\lambda$, questo massimo, si sposta più lentamente al decrescere di $\lambda$ forse come $\lambda^{1}$. Sempre per più alti valori di $\lambda$ il massimo $\chi$ dovrebbe essere dell'ordine di grandezza $\lambda^{1}=k / \chi^{a}$, cioè il prodotto $(\lambda \chi)$ che appare nella [13] per la $g_{k}$ dovrebbe assumere un massimo uguale ad 1.

Al suo massimo, la portata assumerebbe un valore vicino a quello stazionario $\left(q_{k}=k_{e} E / a\right)$. Diminuendo $\lambda$, anche questo massimo decresce ma più lentamente di quanto non si sposti a destra: (riferendoci sempre a $\lambda \psi$, e non a $\psi$ ). 
Per il contributo alla "portata " proveniente dal termine in $(\lambda \varphi)$, esso porta arl un valore tanto più grancle, quanto maggiore $\grave{e} \lambda$,

$$
\left(l i \lambda \frac{H_{\mathrm{o}}-H_{p}}{a}\right)
$$

In generale col decrescere di $\lambda$ tutte le curve, relative alle varie portate, risulteranno piu piatte e piu depresse, in compenso si avvicineranno più lentamente a zero. Infatti l'acqua asportata (rappresentata dall'area racchiusa), leve rimanere la stessa, essenclo lo stato finale $\left(H_{\nu}-E l i c / l\right.$ ) indipendente da $\lambda$.

4) - Verliamo ora l'entità dell'acqua espulsa al catorlo, fino al tempo $\vartheta$ con il dispositivo elettrico chiuso, limitato, (senza tener conto cioè dell'effetto extranorlico).

Ia pressione irlrogeostatica si esprime con la [8], e cioè:

$$
H-H_{p}=\left(H_{0}-H_{p}\right) \varphi+\frac{l}{k} E \psi
$$

$\varphi$ e $\psi$ date in $[2]_{4}:[23]$, serie convergenti, in funzione di $\mu_{n}$, come è noto essendlo $\mu_{n}$ la ennesima $(n=0,1, \ldots)$ soluzione dell'equazione [18]: [3] $\mu$ tan $\mu=\lambda$ (posizioni fondamentali della presente neoteoria).

Se la permeabilità superficiale, si può consirlerare infinita:

$$
\lambda=a_{\chi} \mid k=\infty, \chi=q_{k} /\left(H-H_{p}\right)=\infty, H-H_{p}=0
$$

si avranno le relazioni:

$\mu_{n}=\pi\left(n+\frac{1}{2}\right),(n=0,1,2 \ldots) ;\left(a_{n}=2 / \mu_{n}, b_{n}=2 / \mu^{2}{ }_{n}\right)$,

formule che valgono fino a $\vartheta=\infty$, (se $H_{p}-\frac{l_{e}}{k} E>0$ ).

Per tensioni superiori esse valgono solo fino al momento $\bar{\vartheta}$ in cui la pressione dell'anodlo raggiunge il valore 0 . Il calcolo di questo tempo è un easo particolare del problema del paragrafo 3 ).

Se ora $Q$ ì la quantita totale d'acqua estratta al catorlo, per uniti di sezione del merlesimo fino al tempo $t$, usualmente si calcola 'questa grandezza integrando rispetto a $t$. Nel nostro caso è conveniente pro- 
cedere diversamente, tenendo presente che la quantità d'aequa contenuta nell'unità di volume è data da $(K C H)$, e che la densità iniziale è $\left(H_{0} k \frac{d \varepsilon / d_{p}}{1+\varepsilon}\right)$, si ha per $Q$ :

$$
Q=\frac{l d \mathrm{~d} \varepsilon / \mathrm{d}_{p}}{1+\varepsilon} \int_{0}^{a}\left(H_{\mathrm{o}}-H\right) \mathrm{d} x=l i c \int_{0}^{a}\left(H_{\mathrm{o}}-H\right) \mathrm{d} \cdot \mathrm{d}
$$

Sostituendo nella $[4]_{4}$ la $[4](x=a \xi)$, si ottiene:

$$
\begin{gathered}
\vartheta=h \frac{d \varepsilon / \mathrm{d}_{p}}{1+\varepsilon}\left|\left(H_{\mathrm{o}}-H_{p}\right) f(\vartheta)+\frac{1}{2} \frac{k_{i}}{k} E g(\vartheta)\right|= \\
=k c a\left|\left(H_{\mathrm{o}}-H_{p}\right) f(\vartheta)+\frac{F}{2} \frac{k_{l}}{k} g(\vartheta)\right|
\end{gathered}
$$

ove si è posto:

$$
f=\int_{0}^{1}(1-\varphi) \mathrm{d} \xi, \quad g=-2 \int_{0}^{1} \psi \mathrm{d} \xi
$$

Sostituendo le [2] nelle [6], integrando e ricordando che:

$$
\int_{0}^{1} \cos \mu_{n}(1-\xi) \mathrm{d} \xi=\left(\operatorname{sen} \mu_{n}\right) / \mu_{n}=(-1)^{n} \lambda / \mu \sqrt{\mu_{n}^{2}+\lambda^{2}}
$$

si ha:

$f(\vartheta)=1-\sum_{\vartheta}^{\infty} \alpha_{n} e^{-\mu^{2} n \vartheta} \quad, \quad \zeta(\vartheta)=1-\sum_{\vartheta}^{\infty}(-1)^{n} \beta_{n} e^{-\mu^{2}{ }^{2} \vartheta}$

dove in virtì delle [t]

$$
\begin{gathered}
a_{n}=\frac{2 \lambda^{2}}{\mu^{2}\left(\mu^{2}+\lambda^{2}+\lambda\right)}, \beta_{n}=4 \lambda\left|\sqrt{\lambda^{2}+\mu^{2} n}-(-1)^{n} \mu_{n}\right| \\
\mu^{2}{ }_{n}\left(\mu^{2}+\lambda^{2}+\lambda\right)
\end{gathered}
$$

Per $\lambda=\infty$ le $[8]_{+}$si semplificano (caso standard), come segue:

$$
\alpha_{n}=-2 / \mu^{2}, \quad \beta_{n}=4 / \mu^{3}{ }_{n}
$$

So al posto di $Q$ eonsileriamo il rapporto 0 tra l'aequa estratta e quella inizialmente contenuta nel terreno:

$$
Q=Q /+a c Q_{b}
$$


la [o] diventa:

$$
\varrho=\left(1-\frac{H_{p}}{H_{\mathrm{o}}}\right) f(\vartheta)+\frac{1}{2} \frac{k_{e}}{k} \frac{E}{H_{\mathrm{o}}} g(\vartheta)
$$

un rapporto "indice", caratterizzante il processo e la sua consistenza, (il che impone alcuni pre-riconoscimenti geoidrologici misure di $H_{0}, k, l_{e}, E$, ecc.).

Per $\lambda \rightarrow \infty$ (molto grande) si ha:

$$
\begin{aligned}
\underline{o}_{i=}= & =\left(1-\frac{H_{p}}{H_{0}}\right)\left(1-\sum_{0}^{\infty} \frac{-2}{\mu_{n}} e^{-\mu^{2}{ }^{n} \vartheta}\right)+ \\
& +\frac{1}{2} \frac{k_{e}}{k} \frac{E}{H_{0}}\left(1-\Sigma(-1)^{n} \frac{4 e^{-\mu^{2}{ }^{2} \vartheta}}{\mu^{3} n}\right)
\end{aligned}
$$

Le funzioni $f(\vartheta), g(\vartheta)$, si calcolano mediante le serie $[\bar{\gamma}]_{4}$, che convergono rapidamente, più che non le rispettive $(p, \psi)$. Esse rappresentano funzioni che aumentano da zero per $\vartheta=0$ ad 1 per $\vartheta=\infty$.

Per $H_{p}-\frac{k_{e}}{k} E>\vartheta$. la $[10]_{4}$ vale fino a $\vartheta=\infty$, e ne consegue:

$$
\varrho_{\infty}=1-\frac{H_{p}}{H_{0}} \quad: \frac{1}{2} \frac{k e}{k} \frac{E}{H_{0}}
$$

che rappresenta la massima quantitì d'acqua estraibile, diversamente la

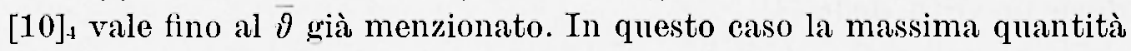
d'acqua che si può estrarre con elettrotensioni che non arriveranno a valori alti, è data da $\varrho_{\infty}=1-\frac{1}{2} \frac{H_{p}}{H_{0}}$.

Passiamo alla valutazione di $\vartheta$ (tempo adimensionale), o della durata in cui viene estratta la frazione d'acqua $\varrho<\varrho_{\infty}, \varrho<\varrho(\vartheta)$, quando $H_{p}-\frac{k_{\epsilon}}{k} E<0$.

Il valore $\vartheta^{\prime}$ si ottiene risolvendo l'equazione [10] $]_{4}$ rispetto a $\vartheta$ : si può procerlere al solito per via grafica o numerica approssimata.

Il $1^{\circ}$ metodo è da preferirsi per valori di $\vartheta$ non troppo alti, il $2^{\circ}$ 
quando $e^{-\pi^{2 \vartheta}}=e^{-1 v \hat{v}}$ è trascurabile rispetto all'unità. Con il metodo grafico, eseguita la tabulazione delle $f(\vartheta), g(\vartheta)$, si traccia la curva:

$$
x=f(\vartheta), \quad y=g(\vartheta)
$$

La curva è tutta contenuta in $0 \leqslant x, y<1$, parte clal punto $(0,0)$, e arriva al punto $(1,1)$.

Disponenclo di questa curra si traccia la retta:

$$
\left(1-\frac{H_{p}}{H_{0}}\right) x+\frac{1}{2} \frac{k_{e}}{k} \frac{E}{H_{o}}=\varrho,
$$

e si determina la sua intersezione con la suddetta curva. Siano $(\bar{x}, y)$ le coordinate del punto d'intersezione; si determina ora, o dal grafico di $f(\vartheta), g(\vartheta)$, o dalle tabelle, il valore comune di $\vartheta$ per cui si ha:

$$
\bar{x}=f(\vartheta), \bar{y}=g(\vartheta)
$$

E più conveniente per quest'ultima determinazione, usare il valore di $g(\theta)$ che aumenta più rapidamente, ar eccezione di valori molto bassi dove ì la $f(\vartheta)$ che cresce più rapidamente.

Si noti che le rette che corrispondono agli stessi valori $\left(H_{0}, F\right)$, e differenti $o$, sono tutte parallele tra di loro, come nel caso del calcolo del tempo di prosciugamento dell'anodo.

Il calcolo numerico approssimato richiede la verifica a posteriori che il $\vartheta$ ottenuto soddlisfi alla condizione che $o^{-10^{\vartheta}}$ sia trascurabile nei limiti della precisazione richiesta in confronto all'unita, (altrimenti il valore ottenuto è al massimo inclicativo). In questo caso nella [7]. si possono trascurare tutti i termini delle $\Sigma$ oltre al $1^{\circ}(n=0)$, porre cioc:

$$
f(\vartheta)=1-\alpha_{0} v^{-\mu^{2} \vartheta} \quad, \quad g(\vartheta)=1-\beta_{0} e^{-\mu_{0}^{2} \vartheta}
$$

Con queste posizioni l'equazione diventa:

$$
\begin{gathered}
{\left[\left(1-\frac{H_{p}}{H_{0}}\right) \alpha_{0}+\frac{1}{2} \frac{k_{e}}{l_{i}} \frac{E}{H_{0}} \beta_{\mathrm{o}}\right] e^{-\mu^{2} \vartheta}=} \\
=1-\frac{H_{p}}{H_{\mathrm{o}}} \div \frac{1}{2} \frac{k_{c}}{l_{i}} \frac{E}{H_{\mathrm{o}}}-\rho
\end{gathered}
$$


che risolta rà il $\vartheta$ cercato:

$$
\vartheta=\mu_{0}^{-2} \lg \frac{\left(H_{0}-H_{p}\right) \alpha_{0}+\frac{1}{\Sigma} \frac{k_{e}}{h} E \beta_{0}}{\left(H_{0}-H_{p}\right)+\frac{1}{2} \frac{l}{\dot{k}} E-\varrho H_{0}}
$$

L'applieabilita di questo procerlimento si può estenclere a valori un pó più piccoli di 9 , conservanulo un ulteriore termine nella [7].., e ponenclo in questa 20 termine $\vartheta$ uguale al valore calcolato merliante la $[14]_{1}$ che ora inclicheremo, per evitare confusioni, con $\bar{\vartheta}$. Si porrà:

$$
f(\vartheta)=1-\alpha_{0} e^{-\mu_{0}^{2} \vartheta}-\alpha_{1} e^{-\mu_{1}^{2} \bar{\vartheta}}, \eta(\vartheta)=1-\beta_{0} e^{-\mu_{0}^{2} \vartheta}-\beta e^{-r_{1}^{2} \bar{a}}\left[\gamma^{\prime \prime}\right]_{4}
$$

Sostituenclo le $\left[\mathrm{f}^{\prime \prime}\right]_{4}$ nelle $[10]_{4}$, e posto:

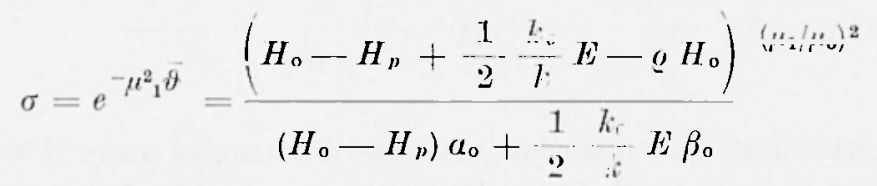

(si noti che o dev'essere tale che risulti $\sigma<1$ ), si perviene all'equazione:

$$
\begin{gathered}
{\left[\left(1-\frac{H_{p}}{H_{0}}\right) \iota_{0}+\frac{1}{2} \cdot \frac{k_{e}}{k_{i}} \frac{E}{H_{0}} \beta_{0}\right] e^{-\mu^{2} \vartheta}=} \\
=\left(1-\frac{H_{p}}{H_{0}}\right)\left(1-\iota_{1} \sigma\right)+\frac{1}{2}-\frac{k_{e}}{k_{i}} \frac{E}{H_{0}}\left(1+\beta_{1} \sigma\right)-\varrho,
\end{gathered}
$$

la eui si ricava il valore del tempo atimensionale:

$$
\vartheta=\mu_{0} \cdot \lg \frac{\left(H_{0}-H_{p}\right) \alpha_{0}+\frac{1}{2}-\frac{l}{h} E \beta_{0}}{\left(H_{0}-H_{p}\right)\left(1-\alpha_{1} \sigma\right)+\frac{1}{2} \frac{l_{e}}{k} E\left(1+\beta_{1} \sigma\right)-\varrho H_{0}}
$$

con $\sigma$ rata rlalla $[15]_{4} ;$ anche la $[15]_{4}$ richierle una verifica a posteriori; ora però non sarì più necessario che $e^{-\pi^{2} \vartheta}$ sia trascurabile, basterà che lo sia $e^{-\dot{4} \pi^{-\hat{v}}}$ in confronto all'unita, nei limiti della precisione richiesta.

La selezione o la classifica dei snoli elettroattivi viene fatta preliminarmente "sul terreno" rilevanclo profili di acqua pompata senza 
corrente elettrica e con corrente a diverse elettrotensione e stesura bipolare). Ha poca importanza l'esame standard di test carotati, potendo esso non significare alcun indice di massa.

Le più intense reazioni $\mathrm{EGO}$ (a parte quelle incisive, ottimali dei suoli coerenti (colloidali), appaiono nelle zone rli repositi serlimentari in corrispondenza dei ritmi rallentati, o nei trapassi d'interstrato, a localizazioni a "squeezings" o digitate, fasce-rlepositi soggette an elettrodiagenesi naturale (effetti Dorn ed altro).

Energizzate dai c.e. si hamno reazioni di massa nelle manovre d'incremento di spaziatura eteropolare, reazioni globalmente talvolta accentuate. Detto scopo si può ragriungere se si impiegano tecniche idonee, e non limitandosi a spaziature fisse polari (definizione di ampiezze polari elettrocinetiche). Con ciò si è studiato l'effetto di un EGO non stazionaria, ritenendo operante al catorlo una permeabilità superficiale finita. Dai risultati ottenuti si comprencle l'importanza della funzione estrattiva, teoricamente finora trascurata, continua, intensa o meno, arl intermittenze di pompagrio e di energizzazione.

I a quantiti d'acqua tellurica estratta e il relativo tempo di estrazione sono stati calcolati in una visione integrale, comprenclente le permeabilità migratorie di volume, idraulica, elettrosmotica, nonchè quella superficiale in corrispondenza delle discontinuitì dei pozzi estrattivi (in gradlo di variare a seconda i pompaggi e i modelli geobipolari). L'attuale impostazione del problema estrattivo porta, come si ̀̀ visto, ar immerliate ripercussioni geotecniche, rigorosamente derlotte, soprattutto sulla neo-possibilita di geoconsolidamenti rapidi e ireversibili, (p.e. in terreni a risentimenti elettrogeocinetici anche minimi). 